\title{
Research on 3D Reconstruction Technology of Tool Wear Area
}

Maohua Xiao, Zhenmin Sun, Xiaojie Shen, Liping Shi, Jing Zhang

College of Engineering, Nanjing Agricultural University, No.40, Dianjiangtai Road, Pukou Distinct, Nanjing210031. China. E-mail: xiaomaohua@njau.edu.cn, 305868962@qq.com, 434355225@qq.com, 125893610@qq.com, zhangjing@njau.edu.cn

The operators' poor adaptability on photos and difficulties of removing background point and noise point and other problems make the conventional focusing synthesis techniques difficult to get better application and promotion in the field of three-dimensional reconstruction. In this paper, a modified Laplacian operator was used as an evaluation basis to select focus points and the theory of three-dimensional reconstruction was discussed from the perspectives of image multilayer composite algorithm and height interpolation. To solve the problem of removing noise point and background point, a double threshold selection technique was proposed, which greatly improved the adaptability of focusing evaluation operator. Finally, a test on three-dimensional reconstruction of the tool wear's surface topography was conducted.

Keywords: Tool wear, Three-dimensional reconstruction, Focusing synthesis techniques, Double threshold

\section{Introduction}

Tool wear does not only affect the accuracy of the workpiece surface, but also has a direct impact on the processing quality of the workpiece[1]. With the improvement of machine vision system, computing hardware and non-tactile application, the digital image processing techniques has become an important role in tool condition monitoring [2,3] .Three-dimensional reconstruction on the region of tool wear based on computer vision technology is a direct monitoring on tool. By reconstructing a three-dimensional information of the tool wear area under the microscope, more information on tool wear surface morphology parameters could be gained, and analysis of the wear mechanisms could be conducted more accurately[4].

In recent years, the research on the use of the microscope and computer vision technology to measure tool wear was getting more popular. HH Shahabi studied Vision-based systems using a CCD camera are being used for measurement of tool wear [5]. Samik Dutta et al performed the micro-scale analysis of turned surface by using discrete wavelet transform[6].Optical three-dimensional reconstruction and measurement methods varied.

Due to operators' poor adaptability on photos, difficulties of removing background point and noise point and other problems, the conventional focusing synthesis techniques have been difficult to get better application and promotion in the field of three-dimensional reconstruction. This paper proposed a method to reconstruct tool wear area by using the principle of focusing synthetic. The authors collected the wear areas' image sequences of different focus depth from a microscope, and through the method of digital image processing, the authors extracted depth information and reconstruct tool wear surface. The first use of dual threshold technology in the field of removing noise point and background point gets good results.

\section{Selection of focusing evaluation function}

Determining if an image is focusing mainly depends on whether the observation image is clear or not, and the same to computer on determining whether the image is focusing. Since the focused image is clearer than defocused image, the focused image contains more information and details, also correspondingly contains more highfrequency components, so whether the image is focusing or not has a great relationship with its high-frequency component. Therefore, one can select appropriate focusing evaluation function to determine the focus position of the microscope [7].

Common focusing evaluation function is mainly divided into two categories, and one is airspace functions and the other is transform domain functions. Airspace functions include gray variance, gray gradient operator, and image gray entropy. Most of the focusing evaluation functions are used in the automatic focusing of the microscope[8]. A comparison and judgment of focus functions value determines the lens focus and therefore the lens height is adjusted. In this paper, the height of the tool wear surface is determined by analyzing the focusing evaluation function and the microscope head height.

Previous gradient gray operator[9] is the first derivative while the second partial derivative can get highfrequency component, arriving at sharpen edges. Laplacian operator is a second derivative operator, which can be treated as the estimated amount of high frequency components. However, for the second partial derivative at $\mathrm{X}$ direction and $\mathrm{Y}$ direction, the sign may be opposite and cancel each other out, hence the focused image shows deviation. Therefore, taken the effectiveness and time of implementation into consideration, point-based improved Laplacian (Modified-Laplacian) operator is chosen to make image superimposed, which is calculated as follows[10]:

$$
\sum\left|\nabla^{2} f(x, y)\right|=\sum\left(\left|\frac{\partial^{2} f(x, y)}{\partial x^{2}}\right|+\left|\frac{\partial^{2} f(x, y)}{\partial y^{2}}\right|\right)
$$

In the formula, $\mathrm{f}(\mathrm{x}, \mathrm{y})$ is gray value of image at the point $(x, y)$.

Generally, differential is replaced by difference to calculate in digital image processing. In conventional 
Laplacian operator image processing, the $3 \times 3$ operator is used, but taking into account the changes in microscopic images texture, the literature[11] proposed an improved Laplacian operator, introducing the use of variable step sizes to calculate second order differential and formula is as follows:

$$
\begin{gathered}
M L(x, y)=|2 f(x, y)-f(x-s, y)-f(x+s, y)| \\
+|2 f(x, y)-f(x, y-s)-f(x, y+s)| \\
L_{0}=\max \sum_{x} \sum_{y} M L(x, y)
\end{gathered}
$$

In the formula, $F(x, y)$ is the focusing value of the point $(x, y)$, representing the focusing measure of the point; $M L(x, y)$ is the Laplacian operator of the image at the point $(x, y) ; s$ is step size, which generally takes 3 , and the point $L_{0}$ is the position of the focusing position.

\section{Focusing Synthesis Technology}

Focused synthesis method involves the acquisition of the microscopic sample sequence images in different object distances such that each image in the whole sequence has a focused clear zone and a focused blurring zone, then the acquisition of the focused clear position corresponding to each pixel in the sequence images according to a certain fusion rule so as to obtain the height index image, and finally the recovery of the depth information based on a certain algorithm. The schematic of focused synthesis is shown in Fig. 1.

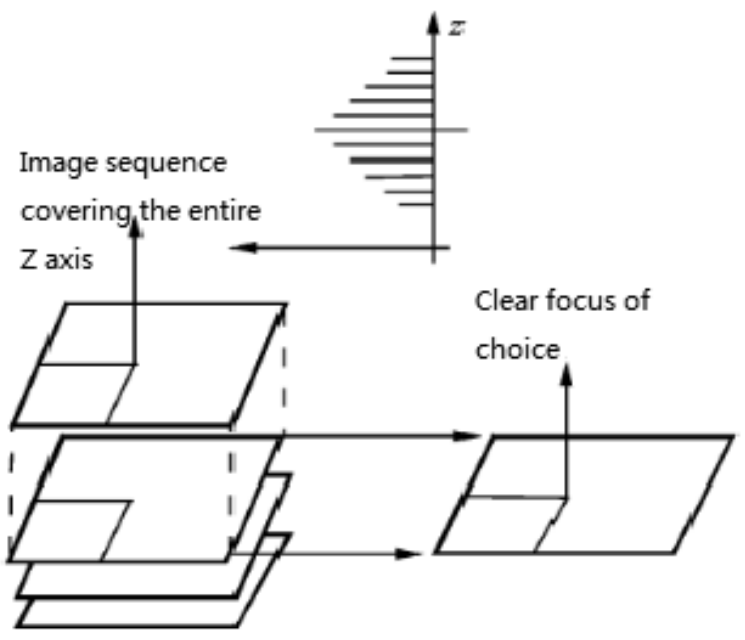

Fig. 1 The schematic of focused synthesis

\subsection{Image multilayer composite algorithm}

In this paper, fine adjustment on the abrasive surface height measurements was applied to adjust the microscope's Z-axis to move equidistantly, and images at each equidistant position were captured, at the same time, the image pixels in Laplacian operator were analysed. A point on the wear surface is getting focusing from defocus during the relative motion processing, and then to defocus, and the image followed by its focus fuzzy to clear, and then from clear to fuzzy. Focusing evaluation function of the position on the abrasive surface follows the focusing process from low to high and then from high to low, where the highest point is the focusing height.
Computational formula of focusing height as follows[12]:

$$
\operatorname{ML}(x, y)=\max (L(x, y, n))
$$

In the formula, $L(x, y, n)$ is improved Laplacian value that is calculated in the nth image at the point $(x, y) ; M L$ $(x, y)$ is the position corresponding to the point's focusing height, whose gray value of the image is the gray value of the point of fusion image and corresponding to the height of the frame is the height of the object point.

After the gray selection and determination of height of each point, information needs to be aggregated in order to obtain gray information and three-dimensional information of the worn surface, and then obtain altitude matrix $M N(x, y)$ :

$$
M N(x, y)=\mathrm{n} L(x, y, n)=M L(x, y)
$$

In the formula, $L(x, y, n)$ is sharpness value gained by Laplace algorithm at coordinate $(x, y)$ of the nth image; $M N(x, y)$ is sequence number of the images at coordinates $(x, y)$ of the focused image, also the height matrix of the wear surface.

After the determination of the height $n$ of each point in the wear area, the grayscale value is determined correspondingly, and the gray matrix $F M L(x, y)$ is:

$$
F M L(x, y)=f(x, y, n) n=M N(x, y)
$$

In the formula, $f(x, y, n)$ is the pixel of height $n$ at the point $(x, y)$, and pixel values at relevant positions are got as the pixel values of the positions.

\subsection{Height Interpolation}

Experiment found that improved Laplacian operator's focusing evaluation presented normal distribution at near maximum value[13], so Gaussian interpolation was employed to estimate the precise focus position of the tool wear surface based on subject's surface height information.

Based on Gaussian interpolation principle, focusing evaluation function can be evaluated in the following formula[14]:

$$
F=F_{p} \exp \left\{-\frac{1}{2}\left(\frac{d-\bar{d}}{\sigma_{F}}\right)\right\}
$$

In the formula, $F$ is the focusing evaluation function; $F_{P}$ is the maximum value of $F$; $d$ is the height.

As is shown in Fig. 2, and according to Gaussian distribution[15]. The natural logarithm is used in the formula, and then the following formula can be obtained.

$$
\ln F=\ln F_{p}-\frac{1}{2}\left(\frac{d-\bar{d}}{\sigma_{F}}\right)^{2}
$$

By replacing each operator's focusing evaluation $F_{m-1}, F_{m}, F_{m+1}$, and appropriately substituting formula (8), we can get three equations, and can be calculated:

$$
\begin{gathered}
\bar{d}=\frac{\left(\ln F_{m}-\ln F_{m+1}\right)\left(d_{m}{ }^{2}-d_{m-1}{ }^{2}\right)}{2 \Delta d\left\{\left(\ln F_{m}-\ln F_{m-1}\right)+\left(\ln F_{m}-\ln F_{m+1}\right)\right\}} \\
-\frac{\left(\ln F_{m}-\ln F_{m-1}\right)\left(d_{m}{ }^{2}-d_{m+1}{ }^{2}\right)}{2 \Delta d\left\{\left(\ln F_{m}-\ln F_{m-1}\right)+\left(\ln F_{m}-\ln F_{m+1}\right)\right\}}
\end{gathered}
$$




$$
\sigma_{\mathrm{F}}^{2}=-\frac{\left(d_{m}{ }^{2}-d_{m-1}^{2}\right)+\left(d_{m}{ }^{2}-d_{m+1}^{2}\right)}{2\left\{\left(\ln F_{m}-\ln F_{m-1}\right)+\left(\ln F_{m}-\ln F_{m+1}\right)\right\}}
$$

Firstly, find $F_{m-1}, F_{m}, F_{m+1}$ that accord to the peak value $F(d)$, and use these evaluation values, to calculate the rough height $d$ in the method of the Gaussian interpolation.

\section{Handling noise and background points with dual threshold selection techniques}

In the actual shooting process, because of many factors, such as affection of the light and hardware devices, image acquisition process certain noises point can cause the Laplacian value suddenly increasing at part of location, thus mistaken image is in the focusing area, causing distortion. On the other hand, the background points' brightness is generally darker, but the Laplace operator is a second order operator that is not sensitive to the area where frequencies is small, thus these areas can not be detected. So Laplacian operator's usable range needs to be filtrated. In this study, the researcher introduces threshold value into the gradient operator to filtrate the usable range of Laplace operator filter by comparing the number of images focusing evaluation function.

Values of $F T$ and $F_{\max }$ are gained according to engineering experience. Under normal circumstances, the noise is not the image whose noise jamming is modest can get a smaller threshold value, while those image sequences whose noise jamming is bigger and the distraction of luminance variation can get a slightly larger threshold value.

Therefore, the height matrix $M N(x, y)$ is improved as shown:

$$
M N(x, y)=n \quad L(x, y, n)=M L(x, y)
$$

Similarly, the gray level matrix $F M L(x, y)$ is improved :

$$
F M L(x, y)=f(x, y, n) \quad n=M N(x, y)
$$

For background point processing is to set a uniform height, usually reference height, such as 0 .

After completion of background processing tasks, the wear surface holes which produced because noise can be removed. And fill and patch the holes to give a height value, here interpolation such as nearest neighbor interpolation and linear interpolation can be chosen to fill.

Use $K(i, j)$ represents whether the point in an image is part of empty area. And 0 indicates that it is part of empty, while 1 indicates that the point does not belong to a hole. Thus a formula can be obtained by filling the hole boundary points $I(i, j)[16]$ :

$$
I(i, j)=\frac{I(i-1, j)+I(i, j-1)+I(i+1, j)+I(i, j+1)}{K(i-1, j)+K(i, j-1)+K(i+1, j)+K(i, j+1)}
$$

And the interpolated of height value as shown[16]:

$$
h(i, j)=\frac{h(i-1, j)+h(i, j-1)+h(i+1, j)+h(i, j+1)}{K(i-1, j)+K(i, j-1)+K(i+1, j)+K(i, j+1)}
$$

\section{Three-dimensional reconstruction test of tool wear area}

\subsection{Test conditions}

Microscope system uses Mitutoyo AT112-220 tool microscope and SONY SSC-DC498P enhanced color camera, and its illuminant uses the German SCHOTT cold light source. Technical parameters of the system are shown in Tab. 1.

Using YW2 cemented carbide welding tool, as shown in Fig. 2.

Tab. 1 System Technical Data

\begin{tabular}{ccccc}
\hline $\begin{array}{c}\text { Measurement } \\
\text { Range }\end{array}$ & Raster Precision & $\begin{array}{c}\text { Size of Imaging } \\
\text { Sensor }\end{array}$ & $\begin{array}{c}\text { Horizontal Resolution } \\
\text { of Imaging Sensor }\end{array}$ & Pixel \\
\hline $200 \mathrm{~mm} \times 100 \mathrm{~mm}$ & $0.001 \mathrm{~mm} / 0.001 \mathrm{~mm} / 0.0005 \mathrm{~mm}$ & $1 / 3$ inches & $480 \mathrm{TV}$ lines & $752(\mathrm{H}) \times 582(\mathrm{~V})$ \\
\hline
\end{tabular}

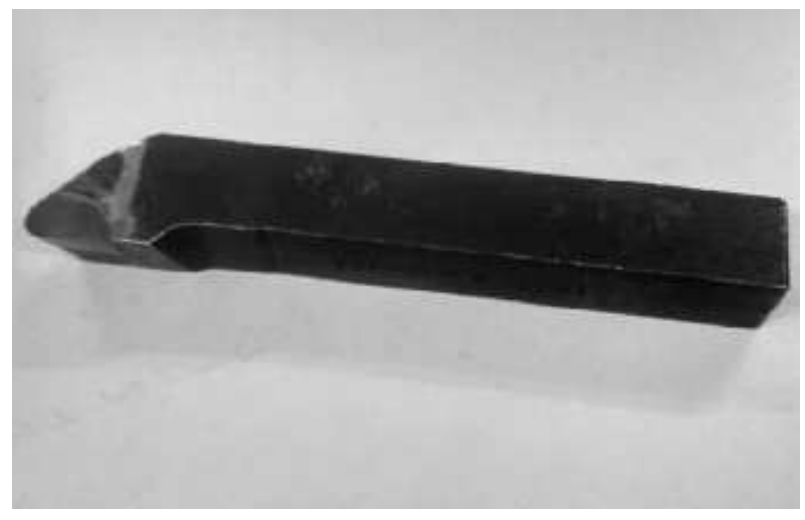

Fig. 2 YW2 cemented carbide welding tool

\subsection{Test conditions}

First, use Laplace gradient operator to conduct convolution on the current image. If convolution of a point's value is greater than the threshold value, it is considered that it is within the focus range, and then retains it, and set flag bit, whose pixel at the frame is taken as reconstructed image's pixels, and height of the frame at this point is taken as a three-dimensional reconstruction point height. If there is still no image greater than threshold value after the point, it can be considered to be the background; the last image's pixel can be taken as value of reconstructed image at that point. Calculating method of the reserved pixels is shown in the formula (15) below. 


$$
F(x, y)=\operatorname{MN}(x, y) \text { with } \operatorname{ML}(x, y)>T
$$

In the formula, $F(x, y)$ is the focusing value; $M N(x, y)$ is sequence number of the images at coordinates $(x, y)$ of the focused image; $M L(x, y)$ is the Laplacian operator of the image at the point $(x, y)$.

All the points of the current frame in focus range can be obtained from the formula, and then use current depth binary numeral to mark all the points of the current frame within a focus range; use the accumulated part binary numeral to mark binary image of all points that has been stored within the depth of field; use accumulated part grayscale to store grayscale of all the points that has been stored in the depth range; use the new binary image to store binary image of new point that is compared with before in the new depth of field; use the new part grayscale to store grayscale of new point that is compared with before in the depth range. Firstly, find out all points that can focus during the shoot through continuous sharpness analysis of image sequences to find out these points, and then reconstruct the point on accumulated part grayscale by the Boolean algebra arithmetic.

Boolean operation formula of the new part is shown in formula (16), which is mainly used to extract out the new image point that is the current frame $A$ more than that portion of the accumulation section $B$.

$$
\{x:(x \notin A \cap B) \cap(x \in A)\}
$$

Find out all the focus point and, the part that does never focus in the sequence is added to the last reconstruction image as background point, and then completed depth-of-field reconstruction image can be obtained that reflected all information and background information within full focus range at this point.

\subsection{Test results and analysis}

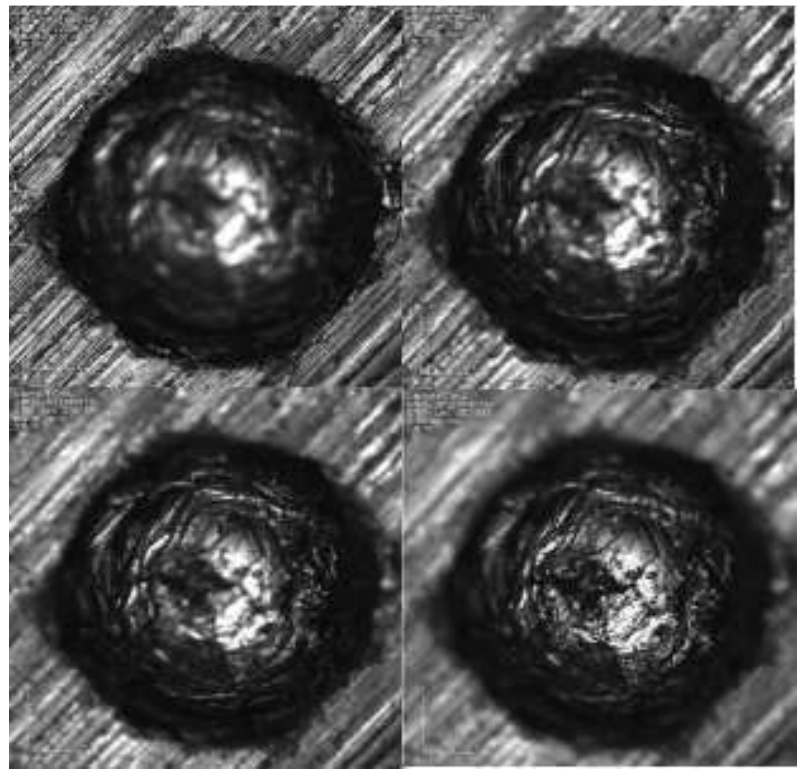

Fig. 3 Four sequential pictures at different focusing height in wear area

Under the same experimental conditions, We observe the wear area of cemented carbide tools. The microscope is adjusted in steps of $0.005 \mathrm{~mm}$, and then make the imaging plane of microscope focus on different height levels in worn area by continuously adjusting the height of the $\mathrm{Z}$-axis of microscope, and then capture sequentially 8frame images in the same area, constitute images sequence, and set the highest surface as the reference surface.

Fig. 3 shows four pictures that are taken when microscope focuses on different heights in wear area. Fig. 4 is the synthesized picture after fusing wear sequence focusing image and extracting the clearest focusing part. Fig. 5 shows the three-dimensional reconstruction of a tool wear area.

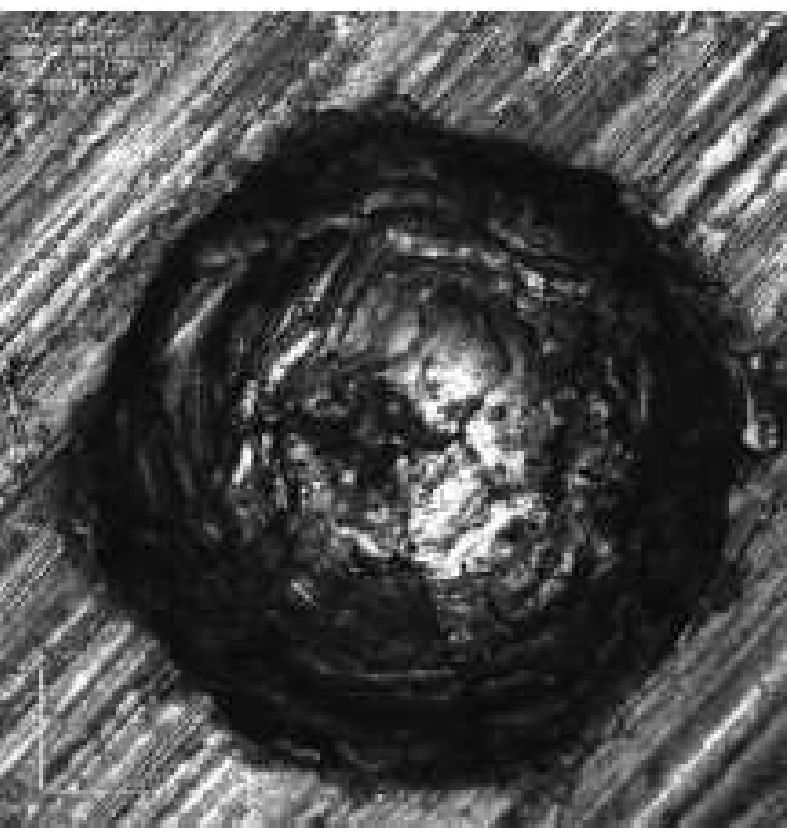

Fig. 4 The whole depth-of-field fusion image in

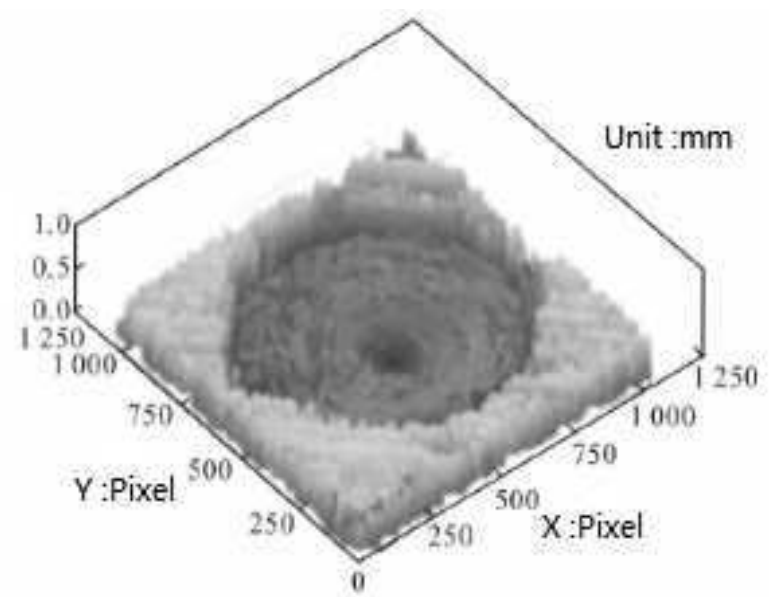

Fig. 5 Three-dimensional reconstruction in wear area

The test is accomplished under the condition of the computer configuration of AMD Athlon 3000+, 512M with Matlab software programming, and the reconstruction time is $122.32 \mathrm{~s}$. A comparison between fig. 4 with fig. 5 shows that three-dimensional reconstruction results do reflect the original appearance of the wear area. 


\section{Conclusions}

(1) Using the methods described herein to conduct experiment, the results of three-dimensional reconstruction can indeed reflect the original appearance of the wear area.The experimental results show the feasibility of the proposed method.

(2) The combination of double threshold and Laplasse operator improves the adaptability of the focusing evaluation operator. It can effectively solve the problem of background points and noise points removal.

(3) Fuzzy area of abrasive middle part stays empty, and the algorithm still needs to be further optimized.

\section{Acknowledgment}

The research is funded partially by the Fundamental Research Funds for the Central Universities fund (KYZ201760) and the Open topic of Jiangsu Provincial Key Laboratory of Advanced Manufacturing Technol$\operatorname{ogy}(\mathrm{HGAMTL-1711).}$

\section{References}

[1] Tomas Baksa, Vaclav Schomik, Pavel Adamek, et al(2018). Effects of Grinding Conditions and Strategy on the Quality of the Cutting Edge. In: Manufacturing Technology, Vol. 18, pp. 3-7. Czech Republic.

[2] DUTTA, S., PAL, S. K., MUKHOPADHYAY, S., et al (2013). Application of digital image processing in tool condition monitoring: A review. In: Journal of Manufacturing Science \& Technology, Vol. 6(3), pp. 212-232. CIRP.

[3] KUCHARIKOVÁ, L., TILLOVÁ, E., BELAN, J., et al. (2016). The Porosity Evaluation with Using Image Analyser Software in Aluminium Cast Alloys. In: Manufacturing Technology, Vol. 16, pp. 989-994. Czech Republic.

[4] NÁPRSTKOVÁ, N., ŠRAMHAUSER, K., CAIS, J., et al. (2018). Using of Electron Microscope to Evaluate the Tool Wear for a Selected Cutting Insert. In: Manufacturing Technology, Vol. 18, pp. 635-640. Czech Republic.

[5] SHAHABI, H. H., RATNAM, M. M. (2009). Incycle monitoring of tool nose wear and surface roughness of turned parts using machine vision. In: International Journal of Advanced Manufacturing Technology, Issue 11-12, pp. 1148-1157. Springer. Germany.

[6] DUTTA, S., PAL, S. K., SEN, R. (2016). Progressive tool flank wear monitoring by applying discrete wavelet transform on turned surface images, In: Measurement, Vol. 77, pp. 388-401. Elsevier. Netherlands.
[7] JIANG ZHIGUO, HAN DONGBING, XIE FENGYING, etc (2004). Chinese stereology and image analysis, In: Chinese Journal of Stereology and Image Analysis, Vol. 9, pp. 31-36. China.

[8] MARTIŠEK, D. (2018). Fast Shape-From-Focus method for 3D object reconstruction. In: Optik-International Journal for Light and Electron Optics, Vol. 169, pp. 16-26. Elsevier. Netherlands.

[9] FARMANULLAH JANA, IMRAN USMAN, et al (2013). Iris localization based on the Hough transform, a radial-gradientoperator, and the graylevel intensity. In: Optik-International Journal for Light and Electron Optics, Vol. 124, pp. 59765985. Elsevier. Netherlands.

[10] ZHAO, H., LI, Q., FENG, H. J. (2018). Multi-focus color image fusion in the HSI space using the sum-modified-laplacian and a coarse edge map. In: Image and Vision Computing, Vol. 26, pp. 12851295. Elsevier. Netherlands.

[11] ZUO, C., LIU, Y., LI, H., et al (2013). Radial stereo imaging system for three-dimensional reconstruction. In: International Journal for Light and Electron Optics, Vol. 124(24), pp. 6700-6706. Optik. Elsevier. Netherlands.

[12] QIU XIAOHUA, LI MIN, ZHANG LIQIONG, YUAN XIANJIE (2019). Guided filter-based multi-focus image fusion through focus region detection. In: Signal Processing: Image Communication, Vol. 72, pp. 35-46. Elsevier. Netherlands.

[13] JIANG, Z. G., SHI, W. H., HAN, D. B., et al (2004). Three-Dimensional Microscopy Image System Based on Depth from Focus. In: Computerized Tomography Theory \& Applications, Vol. 13(4), pp. 9-15.

[14] YAN Q, WENQING Y, XIANGZE L, et al(2014). Variety identification of rice seed based on threedimensional reconstruction method of sequence images. In: Transactions of the Chinese Society of Agricultural Engineering, Vol. 30(7), pp. 190-196. China.

[15] SU NA, FANG JINGLONG (2018). Software Defects Detection Based on Multivariate Guassian Distribution Probability Model. In: Journal of Hangzhou Dianzi University (Natural Sciences), Vol. 38 (5). pp. 34-38. China.

[16] LI JIAFU, TANG WENYAN, WANG JUN, ZHANG XIAOLIN (2018). Multilevel thresholding selecting based on variational mode decomposition for image segmentation. In: Signal Processing, Vol. 147, pp. 80-91. Elsevier. Netherlands. 